www.nature.com/pj

\title{
Effects of UV irradiation on the molecular weight of spider silk
}

\author{
Takashi Matsuhira ${ }^{1}$, Keizo Yamamoto ${ }^{1}$ and Shigeyoshi Osaki ${ }^{2}$
}

The molecular weight of the Nephila clavata spider dragline was determined to be $272 \pm 14$ kDa using a SDS-PAGE method. The effects of ultraviolet (UV) irradiation on the molecular weight of the spider dragline were studied and compared with that of silkworm silk. The band densities observed using SDS-PAGE for the spider dragline and silkworm silk decreased with the UV irradiation time, suggesting decomposition due to the cleavage of protein chain molecules. It was found that the rate constant of degradation due to UV irradiation for the silkworm silk was larger by 1.7 than that for the $N$. clavata spider dragline. This result indicates that the $N$. clavata spider dragline was 1.7 times more resistant to UV irradiation than silkworm silk.

Polymer Journal (2013) 45, 1167-1169; doi:10.1038/pj.2013.41; published online 24 April 2013

Keywords: cleavage; molecular weight; protein molecules; silkworm silk; spider silk; UV irradiation

\section{INTRODUCTION}

Recently, researchers have been interested in spider silk for use in new materials for textile fibers, sutures and violin strings ${ }^{1}$ because of its excellent mechanical, ${ }^{2-5}$ thermal, ${ }^{6}$ aging $^{7}$ and safety ${ }^{8}$ properties. The ultraviolet (UV) resistance of spider silk is one of the important physico-chemical properties of practical textile fibers. It is well known that biomaterials, such as silkworm silk, are very weak with regards to UV irradiation. ${ }^{9}$ In addition, spider silk is expected to be weak with regards to UV irradiation. Previously, Osaki et a $1 .{ }^{10}$ used electron spin resonance to show that the silk of the Nephila clavata spider was more resistant to UV irradiation than that of the Bombyx mori silkworm. Osaki ${ }^{11}$ also found that UV irradiation mechanically strengthened the dragline of the $N$. clavata spider, suggesting the evolution of spiders. ${ }^{12}$ Herein we studied the process of silk degradation due to UV irradiation to clarify why spider silk shows high resistance to UV irradiation. Therefore, we focused on the UV resistance of spider silk from the standpoint of molecular weight, although the molecular weight of $N$. clavata spider silk was undetermined.

In this study, we determined the molecular weight of $N$. clavata spider silk using the SDS-PAGE method and studied the effects of UV irradiation on this spider silk in terms of the molecular weight compared with that of silkworm silk.

\section{MATERIALS AND METHODS}

Sample and UV irradiation

First, spider silk was pulled out of female $N$. clavata spiders (Japanese golden web spider). In addition, silkworm silk (from a cocoon of B. mori) was also employed. UV-A irradiation with a wavelength of $>320 \mathrm{~nm}$ from a Xenon arc lamp (SUNTEST CPS +; Atlas Co., Chicago, IL, USA) was employed to irradiate the silk fibers. The radiation intensity was $700 \mathrm{Wm}^{-2}$. The silk fibers were dissolved in a saturated lithium thiocyanate aqueous solution ${ }^{13}$ to provide a silk concentration of $10 \mathrm{gl}^{-1}$ before and after UV irradiation. After centrifugation of the silk solution, no insoluble precipitates were observed.

\section{Sample preparation}

The solution was dialyzed against an $8 \mathrm{M}$ urea aqueous solution to remove excess lithium thiocyanate electrolytes that interfere with electrophoresis. A 0.188 moll $^{-1}$ Tris- $\mathrm{HCl}$ ( $\mathrm{pH}$ 6.8) buffer containing $30 \%$ sucrose, $6.0 \%$ sodium dodecyl sulfate, $15 \%$ mercaptoethanol and $0.0060 \%$ bromophenol blue was added to the urea-exchanged solution. The ratio of the buffer to the dialyzed silk solution was $1 / 2$ in volume. Next, the prepared silk solutions were incubated for $5 \mathrm{~min}$ at $373 \mathrm{~K}$, and they were used as the silk solution samples for electrophoresis.

High molecular-weight (molecular weight $=220,170,116$ and $76 \mathrm{kDa}$ ) and low molecular-weight (molecular weight $=97 \mathrm{kDa}$ ) Calibration Kits for SDS electrophoresis (Amersham Biosciences UK Ltd., Buckinghamshire, UK), and HiMark Unstained High Molecular Weight Protein Standard (molecular weight $=500,290,240,160,116$, and $97 \mathrm{kDa}$ ) (Invitrogen Co., Carlsbad, CA, USA), were used as molecular-weight markers.

\section{Polyacrylamide gel electrophoresis}

The silk solution for electrophoresis was applied to the 3.0 or $5.0 \%$ polyacrylamide gels ${ }^{14,15}\left(8.5 \times 8.5 \mathrm{~cm}^{2}\right.$ in size $)$, and the electrophoresis was performed at a voltage of $120 \mathrm{~V}$. The proteins in the polyacrylamide gels were stained with Coomassie Brilliant Blue R. The shading of the bands on the polyacrylamide gel films was scanned using an EPSON GT-F650 scanner (Seiko Epson Co., Nagano, Japan). Here the retention factor (Rf) was determined from the migration distance relative to the mobility of the 
proteins in the polyacrylamide gels. The $3.0 \%$ polyacrylamide gel was employed to clearly measure the shades of the bands.

The density of each band was numerically evaluated by integrating the shade in the band area. The correlation curve was obtained from the band densities at a fixed Rf, which was measured for solutions with six different concentrations at which the actual quantity of spider silk protein was known. Therefore, the absolute quantity of molecules at each band was evaluated using a correlation curve. The molecular weight was determined from the $\mathrm{Rf}$ corresponding to the stained protein. We used three types of marker proteins as described above.

\section{RESULTS AND DISCUSSION}

\section{Determination of molecular weight}

Figure 1 shows the band patterns of the 5\% SDS-PAGE for N. clavata spider silk and B. mori silkworm silk. The bands were observed at an Rf value of 0.26 for spider silk and at an Rf of 0.16 for silkworm silk. The molecular-weight measurements were performed for 20 different samples of spider silk. The molecular weight of the $N$. clavata spider silk was determined to be $272 \pm 14 \mathrm{kDa}$ at an $\mathrm{Rf}$ value of 0.26 , which was a little smaller than those $(275-320 \mathrm{kDa})^{13,15-17}$ reported for the dragline silk of other spider species ( $N$. clavipes). The molecular weight of silkworm silk was determined to be $350 \mathrm{kDa}$, which corresponds to the previously reported value (fibroin- $\mathrm{H}, \mathrm{ca}$. $350 \mathrm{kDa}$ ). ${ }^{16,18,19}$

\section{UV degradation of silk proteins}

The effects of UV irradiation on spider silk and silkworm silk characterized in the above section were studied using the band densities in the patterns of 3\% SDS-PAGE to clearly measure the shade density. The band densities in the dried gel film changed with the UV irradiation time. We numerically determined the quantity of molecules with a fixed molecular weight from the band density. However, the band density does not quantitatively reflect the number of molecules because the relationship between the density and the quantity is not necessarily linear. Therefore, we obtained a correlation curve from the band density measured at different quantities of proteins to estimate the absolute quantity of molecules corresponding to the band using the correlation curve.

Figure 2a shows the SDS-PAGE patterns for spider silk after UV irradiation for different periods of time $(0,1,2,3,4$ and $5 \mathrm{~h}$ ). The density of the bands shown in Figure 2a decreased with the UV irradiation time. The results suggest that UV irradiation qualitatively decreased the quantity of silk proteins with a molecular weight of $272 \mathrm{kDa}$.

Figure 3 shows the ratio of uncleaved residue $R$ to initial quantity $I_{0}$ of the spider silk proteins plotted as a function of the UV irradiation time $(t)$. Here, $R$ is equal to $I_{0}$ before UV irradiation, and $I_{0}$ was evaluated from the band density corrected by the correlation curve. The ratio rapidly decreases in the first step and then gradually decreases with the UV irradiation time, indicating that UV irradiation cleaves chemical bonds, thus decomposing the silk chains into fragments with molecular weights $200 \mathrm{kDa}$. In fact, the band density corresponding to the molecular weight of $<20-70 \mathrm{kDa}$ increased with the UV irradiation. Figure $2 \mathrm{~b}$ also shows the patterns of SDS-PAGE for silkworm silk after UV irradiation for different time periods. The band density shown in Figure $2 \mathrm{~b}$ decreases with increasing UV irradiation time $t$. The ratio of the uncleaved residue to the initial quantity of silkworm silk was also plotted as a function of the UV irradiation time, as shown in Figure 3. It was found that the silkworm silk decomposed more easily than the spider silk. Therefore, the spider silk was found to be more resistant to UV irradiation than the silkworm silk.

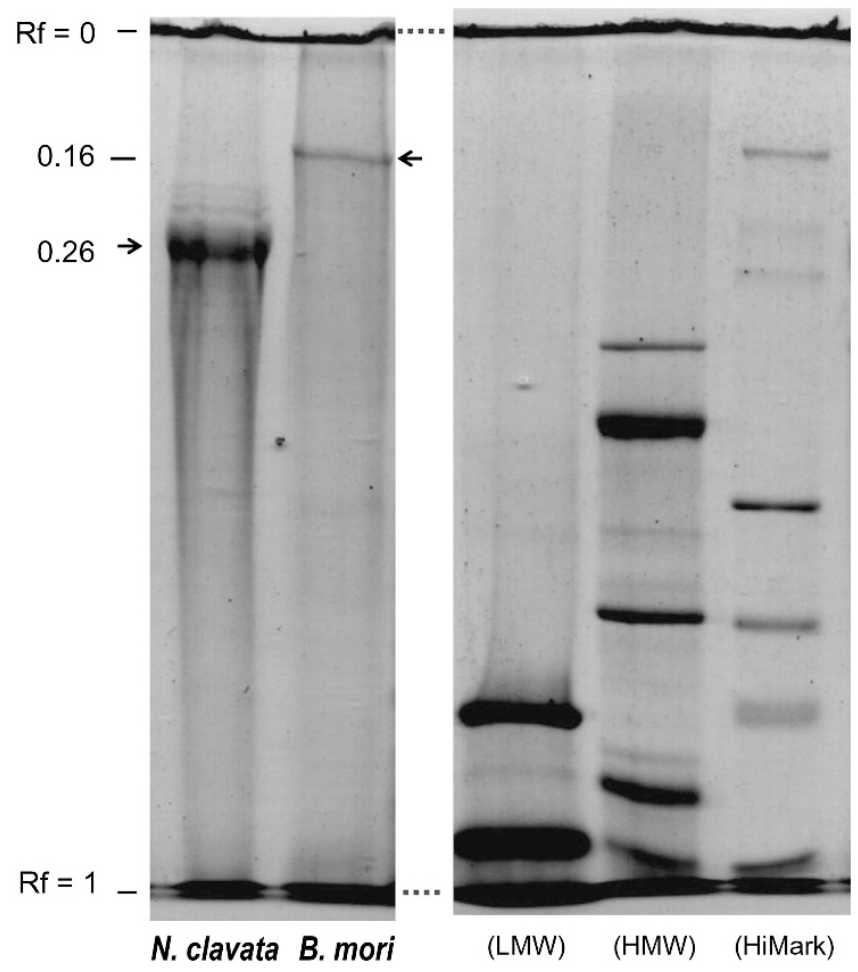

Figure 1 Band patterns of $5 \%$ SDS-PAGE of $N$. clavata spider silk and $B$. mori silkworm silk before UV irradiation. The arrows indicate the bands of silk proteins. For comparison, the bands from three types of molecularweight markers are described on the right side.

Osaki et al. ${ }^{10}$ previously found that the quantity of $\mathrm{C}_{\alpha}$-centered radicals from the cleavage of protein chains induced by UV irradiation for $N$. clavata spider silk was lower than that for B. mori silkworm silk, which indicated that the spider silk is more resistant to UV irradiation than the silkworm silk. Based on the molecular weight, these results strongly support previous results ${ }^{10}$ with respect to the radical quantity.

To numerically compare the degree of resistance to UV irradiation, we studied the relationship between $\log \left(R / I_{0}\right)$ and the UV irradiation time $(t)$ for spider silk and silkworm silk. Here, $R$ is the quantity of uncleaved proteins, and $I_{0}$ is the initial quantity of proteins. The empirical equation was obtained as $\log \left(R / I_{0}\right)=-(1 / 2.303) k t$, where $k$ is the rate constant of degradation indicating the speed of degradation due to UV irradiation. $k$ was determined to be $1.02 \times 10^{-4} \mathrm{~s}^{-1}$ for spider silk and $1.75 \times 10^{-4} \mathrm{~s}^{-1}$ for silkworm silk. This result indicates that spider silk is 1.7 times more resistant to UV irradiation than silkworm silk.

The degradation process is described below. UV irradiation leads to the cleavage of chemical bonds in the protein chains. The cleavage creates radicals that induce the cleavage of other chemical bonds in the same chains as well as in other chains. Therefore, UV irradiation amplifies the number of radicals. These amplified radicals are expected to further accelerate the cleavage of chemical bonds in the protein chains, which then decomposes the spider silk chains into fragments. Thus, the quantity of spider silk chains with a fixed molecular weight decreases exponentially with the UV irradiation time.

Finally, the effects of UV irradiation on the molecular weight indicate that the $N$. clavata spider silk is more resistant to UV 
a

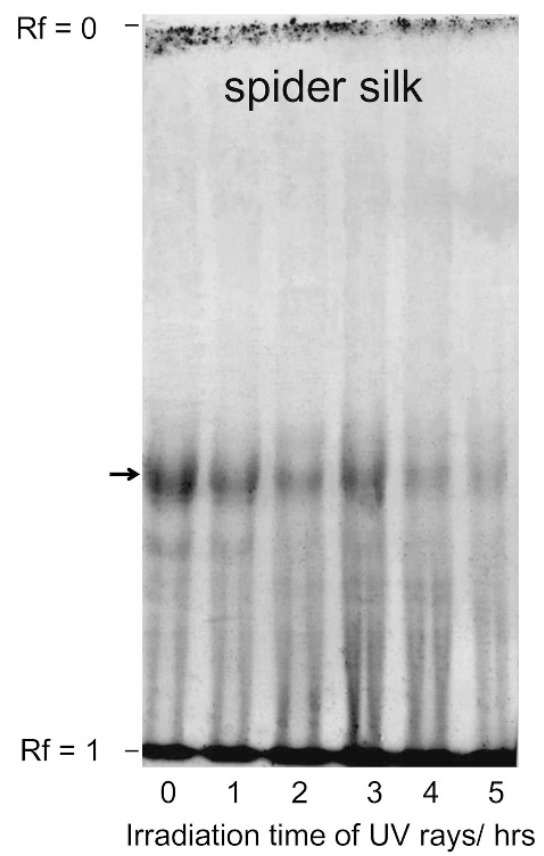

\section{b}

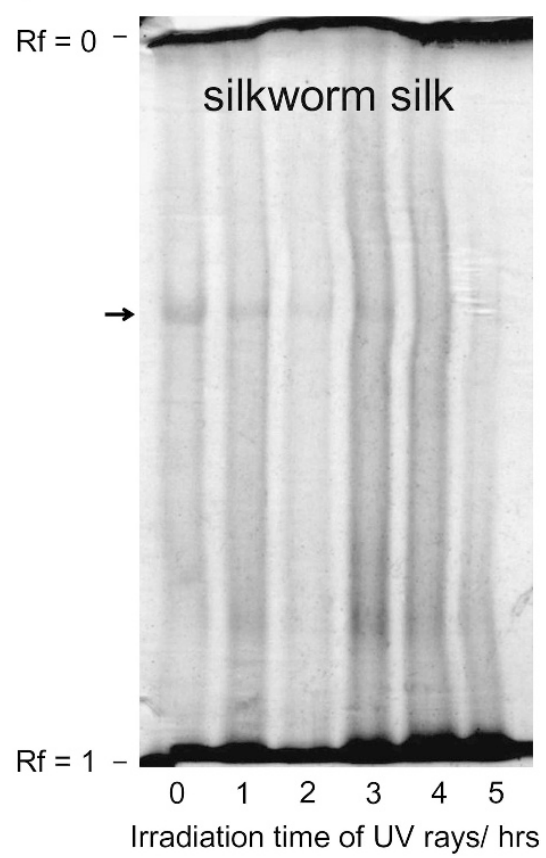

Figure 2 Band patterns for 3\% SDS-PAGE after UV irradiation for different time periods (0, 1, 2, 3, 4 and 5 h). (a) N. clavata spider silk. (b) B. mori silkworm silk.

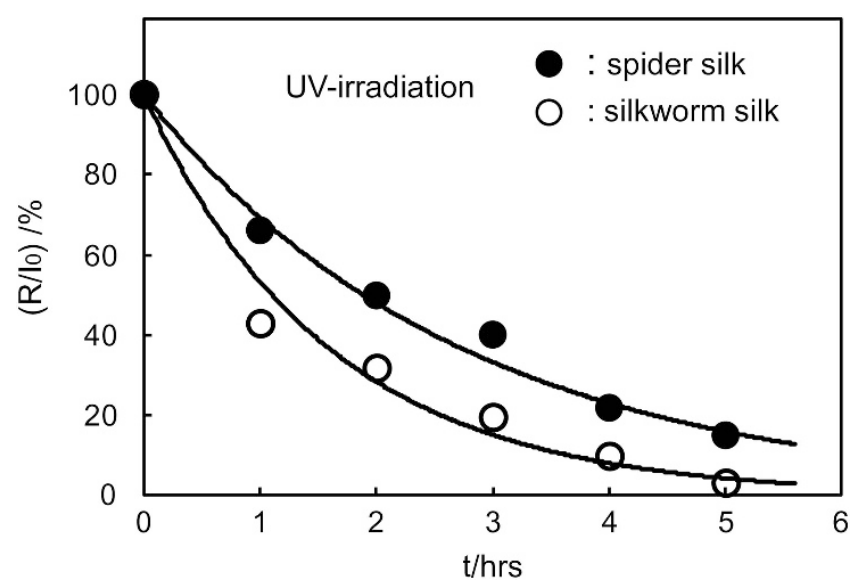

Figure 3 The ratio of the quantity $R$ of residue proteins to the initial quantity $I_{0}$ of proteins is plotted as a function of the UV irradiation time $t$. Black circles: spider silk and white circles: silkworm silk.

irradiation than the silkworm silk, which supports previous results on the radical quantity. ${ }^{10}$ The difference in the UV resistance between spider silk and silkworm silk may be due to several factors, such as the amino-acid sequence or the fine structures, including the $\beta$-sheet structure and molecular orientation.

In the near future, the authors will report how UV irradiation mechanically strengthens spider silk from the standpoint of molecular weight.
1 Osaki, S. Spider silk violin strings with a unique packing structure generate a soft and profound timbre. Phys. Rev. Lett. 108, 154301-154305 (2012).

2 Work, R. W. \& Morosoff, N. S. A physico-chemical study of the supercontraction of spider major ampulate silk fibers. Tex. Res. J. 52, 349-356 (1982).

3 Gosline, J. M., Denny, M. W. \& DeMond, M. E. Spider silk as rubber. Nature 309, 551-552 (1984).

4 Vollrath, F. Biology of spider silk. Int. J. Biol. Macromol. 24, 81-88 (1999).

5 Osaki, S. \& Ishikawa, R. Determination of elastic modulus of spider's silks. Polym. J. 34, 25-29 (2002).

6 Osaki, S. Thermal properties of spider's thread. Acta Arachnol. 7, 69-75 (1989).

7 Osaki, S. Ageing of spider silks. Acta Arachnol. 43, 1-4 (1994).

8 Osaki, S. Spider silk as mechanical lifeline. Nature 384, 419-419 (1996).

9 Hirabayashi, K., Yanagi, Y., Kawakami, S., Okuyama, K. \& Hu, W. Degradation of silk fibrin. J. Seric. Sci. Jpn 56, 18-22 (1987).

10 Osaki, S. Yamamoto, K., Kajiwara, A. \& Murata, M. Evaluation of the resistance of spider silk to ultraviolet irradiation. Polym. J. 36, 623-627 (2004).

11 Osaki, S. Ultraviolet rays mechanically strengthen spider's silk. Polym. J. 36, 657-660 (2004).

12 Osaki, S. \& Osaki, M. Evolution of spiders from noctural to diurnal gave spider silks mechanical resistamnce against UV irradiation. Polym. J. 43, 200-204 (2011).

13 Sponner, A., Schlott, B., Vollrath, F., Unger, E., Grosse, F. \& Weisshart, K. Characterization of the protein components of Nephila clavipes dragline silk. Biochemistry 44, 4727-4736 (2005).

14 Laemmli, U. K. Cleavage of structural proteins during the assembly of the head of bacteriophage T4. Nature 227, 680-685 (1970).

15 Mello, C. M., Senecal, K., Yeung, B., Vouros, P. \& Kaplan, D. in Silk Polymers. Material Science and Biotechnology (eds Kaplan, D., Adams, W. W., Farmer, B. \& Viney, C.) 67-79 (American Chemical Society, Washington, USA, 1994).

16 Hakimi, O., Knight, D. P., Vollrath, F. \& Vadgama, P. Spider and mulberry silkworm silks as compatible biomaterials. Compos. Pt. B: Eng. 38, 324-337 (2007).

17 Vollrath, F. \& Knight, D. P. Liquid crystalline spinning of spider silk. Nature $\mathbf{4 1 0}$ $541-548$ (2001).

18 Takasu, Y., Yamada, H. \& Tsubouchi, K. Isolation of three main sericin components from the cocoon of the silkworm Bombyx mori. Biosci. Biotechnol. Biochem. 66, 2715-2718 (2002).

19 Chengjie, F., Zhengzhong, S. \& Vollrath, F. Animal silks: their structures, properties and artificial production. Chem. Commun. 6515-6529 (2009). 